\title{
Position Tolerance Design Method for Array Antenna in Internet of Things
}

\author{
Congsi Wang $\left(\mathbb{D},{ }^{1,2}\right.$ Shuai Yuan $\left(\mathbb{D},{ }^{1}\right.$ Xiaodong Yang $\left(\mathbb{D},{ }^{3}\right.$ Wei Gao, ${ }^{2}$ Cheng Zhu, ${ }^{4}$ \\ Zhihai Wang, ${ }^{5}$ Shaoxi Wang ${ }^{\circ},{ }^{6}$ and Xuelin Peng ${ }^{7}$ \\ ${ }^{1}$ Key Laboratory of Electronic Equipment Structure Design, Ministry of Education, Xidian University, Xian 710071, China \\ ${ }^{2}$ School of Civil and Environmental Engineering, University of New South Wales, Sydney, NSW 2052, Australia \\ ${ }^{3}$ School of Electronic Engineering, Xidian University, Xian 710071, China \\ ${ }^{4}$ National Key Laboratory of Antennas and Microwave Technology, Xidian University, Xian 710071, China \\ ${ }^{5}$ CETC No. 38 Research Institute, Hefei 230088, China \\ ${ }^{6}$ School of Software and Microelectronics, Northwestern Polytechnical University, Xi'an 710072, China \\ ${ }^{7}$ Nanjing Research Institute of Electronics Technology, Nanjing 210039, China
}

Correspondence should be addressed to Shuai Yuan; xduyuanshuai@126.com

Received 27 October 2017; Accepted 4 January 2018; Published 12 February 2018

Academic Editor: Farooq A. Tahir

Copyright (c) 2018 Congsi Wang et al. This is an open access article distributed under the Creative Commons Attribution License, which permits unrestricted use, distribution, and reproduction in any medium, provided the original work is properly cited.

The position error of array antenna significantly deteriorates the gain and sidelobe of the array, which seriously hinders the realization of high performance of communication antenna for Internet of Things (IoT). Based on the sensitivity analysis theory, the sensitivity of the array radiation field with respect to the position of the antenna element is derived. Besides, a novel design method of position tolerance for array antenna is proposed and applied to a $20 \times 20$ planar array. Compared with the array designed by traditional method, the gain loss is basically the same (being $0.5 \mathrm{~dB}$ ), while the peak sidelobe level is lowered by $1.937 \mathrm{~dB}$ $\left(\varphi=0^{\circ}\right) / 1.586 \mathrm{~dB}\left(\varphi=90^{\circ}\right)$. Besides, the uncertainty analysis results show that the newly designed array has a much higher chance to achieve the desired performance, which fully demonstrates the innovation and effectiveness of the new method.

\section{Introduction}

The Internet of Things (IoT) has received considerable attention in many areas like smart cities [1], health care [2], and so on [3-6]. The array antenna is a key component to acquire the target information in the Internet of Things [7]. In the complicated electromagnetic jamming condition caused by a large number of other objects, the high gain and low sidelobe of array antenna are crucial parameters for the accurate acquisition of the targets' state $[8,9]$. However, affected by the fabrication accuracy and temperature load, the actual position of array element is inevitably deviated from its nominal position, resulting in a significant decrease in the gain and a severe increase in the side lobes [10-16]. This paper focusses on the design method of the position tolerance of antenna element.
There have been a large number of studies focusing on the tolerance design of antenna element. The tolerance of the amplitude and phase error of the excitation is first studied. Ruze [17] first studied the effect of random amplitude and phase error on the mean value of gain and sidelobe level (SLL) from the statistical point of view. Hsiao [18] made a further step and studied the effect of random amplitude error on the peak sidelobe level (PSLL). Besides, the interval method is adopted to analyze the effect of random amplitude/phase error whose probability distribution is unknown $[19,20]$. Besides, with the development of the studies on the position tolerance of amplitude and phase error, the tolerance of the position error of antenna element also receives a lot of attention. Bailin and Ehrlich [21] studied the effect of errors in slot lengths and position on the performance of the slot array. Wang [22] deduced the relationship between 
the position tolerance of antenna element and the gain loss of the array. Elliott [23] proposed the method to design the position tolerance according to the allowable PSLL increasement. Lanne [24] studied the influence of the position error tolerance of subarray. Besides, the effect of random and deterministic position error on the performance of the array is also analyzed $[25,26]$. One thing in common with these tolerance design methods is that in order to meet the requirements of array performance, the tolerance of all elements should be less than the same standard, called the "traditional method" in this paper. However, it is obvious from the qualitative theoretical analysis that the influence of an array element on the electrical performance of array varies from others. If the tolerance of array elements which have larger influence is strictly controlled and that of the array elements which have small influence is reasonably increased, then the performance of the array should be more easier to be satisfied than the "traditional method." Therefore, in order to provide the position tolerance design method of antenna element, how to assess the influence of the position of antenna element on the array performance becomes a crucial problem, which is not yet seen in the public literature.

In this paper, the sensitivity of the main lobe of the array radiation field to the position of antenna element is taken as the parameter to evaluate the influence of the gain on the position of the array, and the corresponding computational model is derived. Besides, the position tolerance of a $20 \times 20$ planar array is designed based on the positional sensitivity. The results show that compared with the array designed by the "traditional method" the gain loss of the newly designed array is basically the same as the "traditional" one, whereas the PSLL is much lower, which proves the validity and innovation of the new method.

\section{Mathematical Derivation of the Sensitivity Model}

The sensitivity analysis can be used to evaluate the influence of independent variables on dependent variable [27] and has been widely used in various fields [28-30], but its application in array antenna design is not found in the public literature. Therefore, it is necessary to derive the sensitivity of the array radiation field to the element position. For a planar array as is shown in Figure 1, the radiation field is $E$ as follows:

$$
E=\sum_{m=0}^{M-1} \sum_{n=0}^{N-1} I_{m n} \cdot \exp \left[j k\left(m d_{x} \mu+n d_{y} v+z_{m n} \omega\right)\right]
$$

The sensitivity $S_{m n}$ of the array radiation field $E$ to the position of the antenna element $(m, n)$ is in fact calculated by the partial derivative. The greater the modulus of the partial derivative, the higher the position sensitivity of the element $(m, n)$, so the position tolerance of the element should be smaller. Besides, it is obvious that (1) satisfies the conditions of derivable function [31]. In addition, it has been proved that compared to the position error in $x, y$ direction the position error in $z$ direction causes a much larger gain loss and sidelobe level [26]. Therefore, the sensitivity of the array radiation field to the position error in $z$ direction is

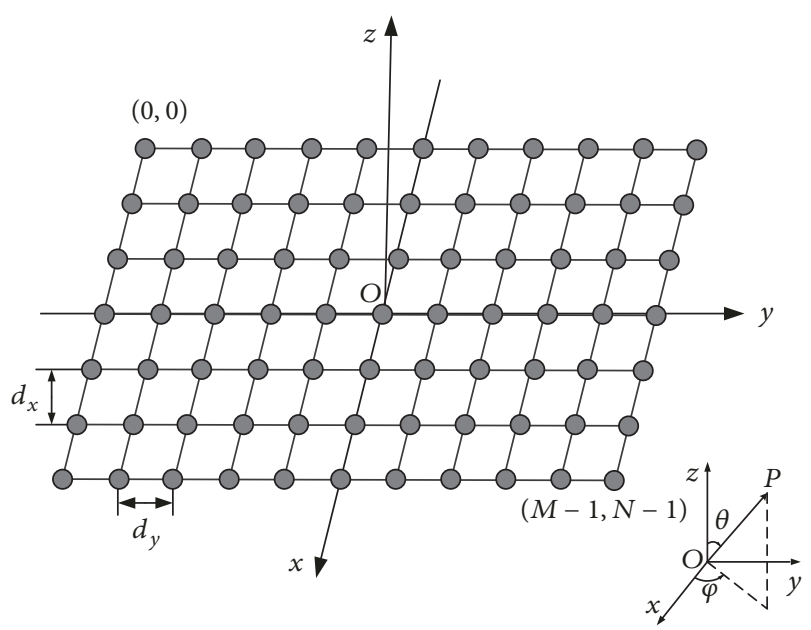

FIGURE 1: Spatial geometric distribution of array antennas.

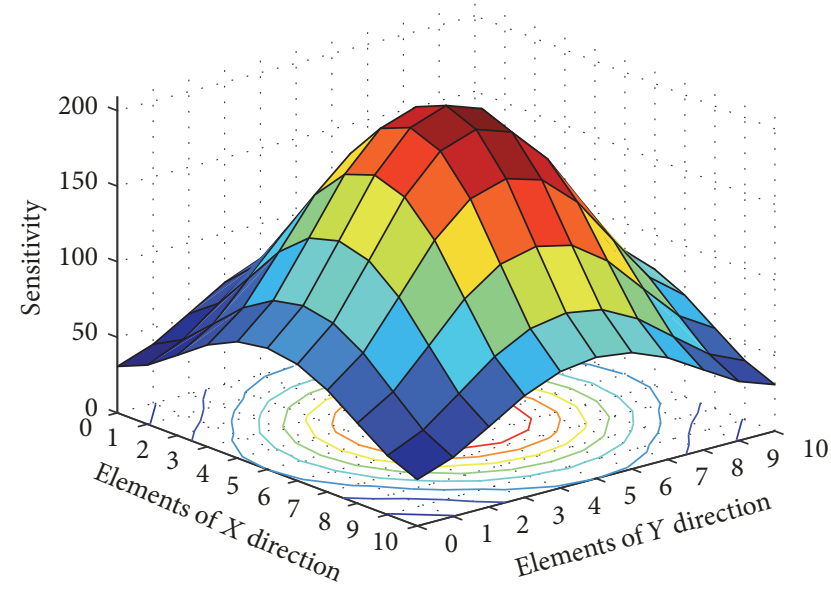

Figure 2: Position sensitivity distribution of array elements.

TABLE 1: Parameters of the array.

\begin{tabular}{cccccc}
\hline$M$ & $N$ & Freq & $d_{x}$ & $d_{y}$ & Weight \\
\hline 11 & 11 & $10 \mathrm{GHz}$ & $0.65 \lambda$ & $0.65 \lambda$ & $\begin{array}{c}\text { Taylor } \\
(-25 \mathrm{~dB})\end{array}$ \\
\hline
\end{tabular}

analyzed in this paper, and the result can be easily extended to the analysis of positions in $x, y$ direction. The $S_{m n}$ can be calculated by the following equation:

$$
\begin{aligned}
S_{m n} & =\frac{\partial E}{\partial z_{m n}} \\
& =j k \omega \cdot I_{m n} \exp \left[j k\left(m d_{x} \mu+n d_{y} \nu+z_{m n} \omega\right)\right] .
\end{aligned}
$$

Taking a $11 \times 11$ planar array as an example, the sensitivity $S_{m n}$ is calculated and is shown in Figure 2. The structural and electrical parameters of the array are shown in Table 1.

It can be seen from Figure 2 that the highest position sensitivity is located in the central region of the array, and the lowest is located in the edge region. Based on the previous analysis, in order to meet the performance requirements, the 


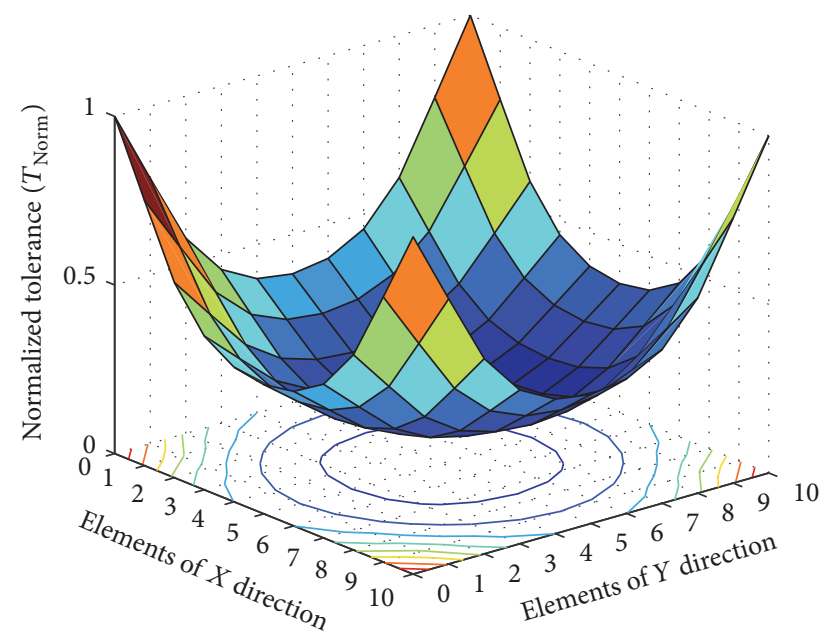

FIGURE 3: Normalized position tolerance distribution $\mathbf{T}_{\text {norm }}$.

higher the position sensitivity is, the smaller the position tolerance should be. Therefore, it is assumed that the position tolerance of each antenna element is positively linearly correlated to the reciprocal of the corresponding sensitivity. Here $\mathbf{T}_{\text {norm }}$ is defined as the normalized position tolerance distribution and calculated as follows:

$$
\begin{aligned}
\mathbf{T}_{\text {norm }}^{m n}= & \frac{\left(1 / S_{m n}\right)}{\max \left(1 / S_{i j}\right)} \\
& (i=0,1, \ldots, M-1 ; j=0,1, \ldots, N-1) .
\end{aligned}
$$

In this case, the normalized position tolerance distribution $\mathbf{T}_{\text {norm }}$ is shown in Figure 3 . Then the position tolerance distribution of the array can be written as $\mathbf{T}=T_{\max } \cdot \mathbf{T}_{\text {norm }}$, where $T_{\max }$ is the maximum position tolerance of all the array elements. Suppose the acceptable gain loss caused by the random position error is $\mathrm{GL}_{\max }$, then how to determine $T_{\max }$ according to $\mathrm{GL}_{\max }$ becomes a critical issue, which is studied in the next section.

\section{Position Tolerance Design Method and Verification}

3.1. Process for Position Tolerance Design. The very first point that needs to be noted here is that the position tolerance $T_{\max }$ is a definite value, whereas the position error of antenna element is randomly distributed within the tolerance zone $\mathbf{T}$, so the gain loss of the array under $\mathbf{T}$ is uncertain, too. In order to quantitatively evaluate the gain loss caused by the position errors that submitted to $T$, the reliable gain loss (R-GL) is defined, which means there is a very small possibility that the gain loss under $T$ is greater than R-GL. Exactly speaking, suppose $X$ groups of position error samples are generated under $T$ and there are $X$ gain losses accordingly. Then $\mathrm{R}-\mathrm{GL}$ is the smallest gain loss of those that ranks in the top $5 \%$ of all gain loss. The definition is also suitable for reliable PSLL (R-PSLL). It is important to note that both R-GL and R-PSLL are directly related to the groups of position

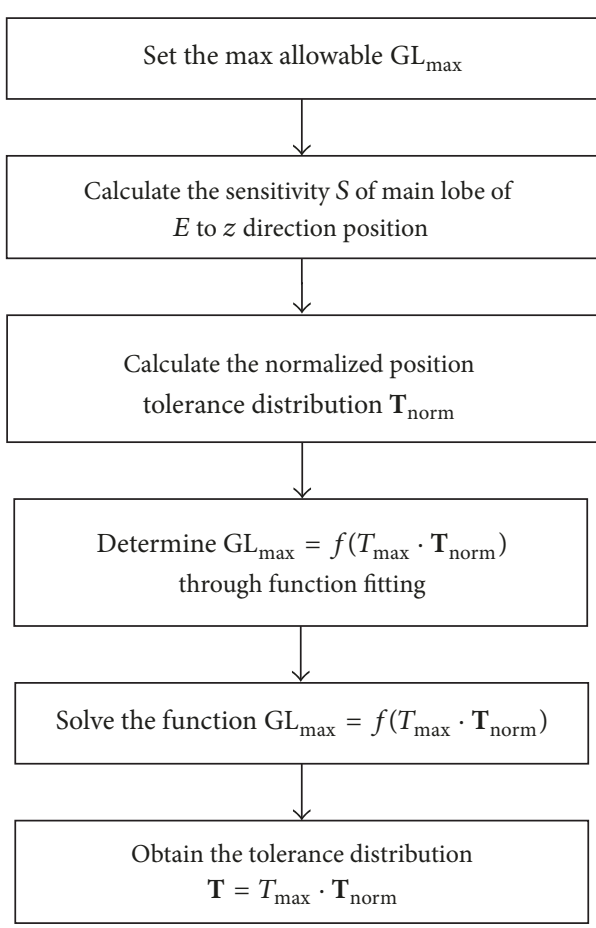

Figure 4: Flow chart for determining the tolerance distribution $T$.

error samples generated, that is, $X$. The bigger $X$, the smaller the variation range of $\mathrm{R}-\mathrm{GL} / \mathrm{R}-\mathrm{PSLL}$. When the variation range of R-GL/R-PSLL is within an acceptable precision, $X$ is acceptable and called the reliable group number $X_{r}$.

Meanwhile, it is known from previous studies that there exists a certain functional relation between the reliable gain loss R-GL [15] and the position tolerance distribution $T$. Suppose $\mathrm{GL}_{\max }=0.5 \mathrm{~dB}$, then the maximum position tolerance $T_{\max }$ can be obtained by directly solving the function $\mathrm{GL}_{\max }=f\left(T_{\max } \cdot \mathbf{T}_{\text {norm }}\right)$. Therefore, in order to obtain $T_{\max }$, it is necessary to find the functional relationship $f$, which can be carried out through the following steps:

(a) Calculate the normalized position tolerance distribution $\mathbf{T}_{\text {norm }}$ through (3).

(b) Generate a series of tolerances $T_{\max }^{t}$ randomly and calculate $\mathrm{RGL}_{t}$ accordingly.

(c) Select the fitting function according to the distribution trend of $\mathrm{RGL}_{t}$ relative to $T_{\max }^{t}$.

(d) Perform the function fitting in MATLAB to get the function $\mathrm{RGL}=f\left(T_{\max } \cdot \mathbf{T}_{\text {norm }}\right)$.

(e) Solve the function $\mathrm{GL}_{\max }=f\left(T_{\max } \cdot \mathbf{T}_{\text {norm }}\right)$; then the corresponding maximum position tolerance $T_{\max }$ is obtained.

In summary, the position tolerance allocation of array antenna is determined as shown in Figure 4.

3.2. Verification of Design Method. In order to embody the advantage of the position tolerance design method in this paper, it is necessary to compare the electrical performance parameters like gain loss and sidelobe level of array designed by the method proposed in this paper and that of the array designed by the "traditional method." For the convenience of 
TABLE 2: Parameters of the array.

\begin{tabular}{cccccc}
\hline$M$ & $N$ & Freq & $d_{x}$ & $d_{y}$ & Weight \\
\hline 20 & 20 & $10 \mathrm{G} \mathrm{Hz}$ & $0.65 \lambda$ & $0.65 \lambda$ & $\begin{array}{c}\text { Taylor } \\
(-25 \mathrm{~dB})\end{array}$ \\
\hline
\end{tabular}

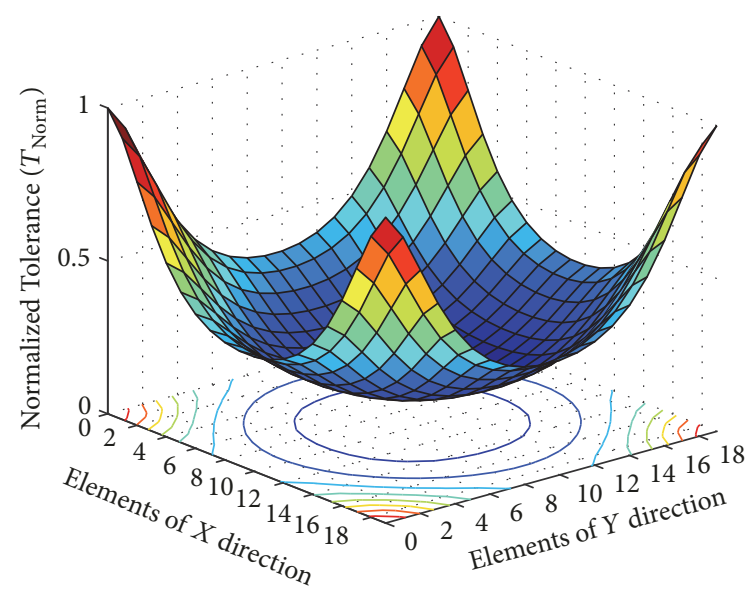

FIgURE 5: Normalized position tolerance distribution $\mathbf{T}_{\text {norm }}$.

narration, the previous array is denoted as SA and the latter one as UA.

The following are the verification process and parameter settings. In order to meet the requirements for antielectromagnetic interference capability of most IoT array antennas, a $20 \times 20$ planar array is taken as an example to illustrate the comparison. The structural parameters and electrical parameters are shown in Table 2, and the normalized position tolerance distribution $\mathbf{T}_{\text {norm }}$ is shown in Figure 5 . The performance of SA and UA is compared under the same allowable requirements. Specifically speaking, the relationship between the GL and the position tolerance $\mathbf{T}$ for SA is obtained through the new method $\left(\mathrm{GL}^{\mathrm{S}}=f(\mathrm{~T})\right)$, and the relationship for UA $\left(\mathrm{GL}^{\mathrm{U}}=g(\mathrm{~T})\right)$ is obtained from Wang [22] that is frequently cited by relevant researchers. Then suppose the allowable gain loss for SA and UA both is $\mathrm{GL}_{\mathrm{a}}^{\mathrm{S}}=\mathrm{GL}_{\mathrm{a}}^{\mathrm{U}}=$ $0.5 \mathrm{~dB}$. The position tolerance for $\mathrm{SA}\left(\mathrm{T}_{\mathrm{a}}^{\mathrm{S}}\right)$ and $\mathrm{UA}\left(\mathrm{T}_{\mathrm{a}}^{\mathrm{U}}\right)$ is obtained through $\mathrm{GL}_{\mathrm{a}}^{\mathrm{S}}=f(\mathrm{~T})$ and $\mathrm{GL}_{\mathrm{a}}^{\mathrm{U}}=g(\mathrm{~T})$, respectively. Finally $10^{\wedge} 4$ sets of position error samples of antenna element are generated under $T_{a}^{S}$ and $T_{a}^{U}$, respectively. The corresponding R-GL and R-PSLL of SA and UA are calculated based on these position error samples. Besides, the uncertainty analysis based on the tolerance distribution of SA and UA is also performed for the comprehensive view of the result. The mean and the standard deviation of the corresponding electrical performance are calculated. What is more, the ratio of the gain loss that is less than $0.5 \mathrm{~dB}$ $(\mathrm{GL} \leq 0.5 \mathrm{~dB})$ and the ratio of the $\triangle \mathrm{PSLL}$ that is less than $1 \mathrm{~dB}(\triangle \mathrm{PSLL} \leq 1 \mathrm{~dB})$ are counted. The reliable group number $X_{r}$ is obtained through the theory of minimum sample size in mathematics of statistics [32] and numerical simulation. When the variation range of R-GL is $0.05 \mathrm{~dB}$, the reliable group number is $X_{r}=100$. Furthermore, the uncertainty of
TABLE 3: Comparison of electrical performance parameters between UA and SA (unit: $\mathrm{dB}$ ).

\begin{tabular}{lcccc}
\hline & R-GL & $\begin{array}{c}\text { R-PSLL } \\
\left(\varphi=0^{\circ}\right)\end{array}$ & $\begin{array}{c}\text { R-PSLL } \\
\left(\varphi=90^{\circ}\right)\end{array}$ & Tolerance \\
\hline SA & 0.502 & -24.442 & -24.328 & $0.023 \lambda \sim 0.163 \lambda$ \\
UA & 0.491 & -22.505 & -22.742 & $0.053 \lambda$ \\
$\begin{array}{l}\text { (Ref. [22] }) \\
\text { Difference }\end{array}$ & -0.011 & -1.937 & -1.586 & $/$ \\
\hline
\end{tabular}

Here the difference is the parameter of SA minus that of UA.

TABLE 4: Uncertainty analysis of SA and UA under the precondition of equal gain loss.

\begin{tabular}{lcccc}
\hline & & Mean & Std. & Ratio \\
\hline $\begin{array}{l}\text { Gain } \\
\text { loss }\end{array}$ & $\begin{array}{c}\text { SA } \\
\text { UA } \\
\text { (Ref. [22]) }\end{array}$ & 0.433 & 0.034 & $97.2 \%$ \\
\hline $\begin{array}{l}\text { PSLL } \\
\left(\varphi=0^{\circ}\right)\end{array}$ & SA & -25.616 & 0.697 & $96.0 \%$ \\
\hline $\begin{array}{l}\text { PSLL } \\
\left(\varphi=90^{\circ}\right)\end{array}$ & $\begin{array}{c}\text { UA } \\
\text { SA } 22])\end{array}$ & -24.214 & 0.721 & $49.5 \%$ \\
\hline & $\begin{array}{c}\text { UA } \\
\text { (Ref. [22]) }\end{array}$ & -24.201 & 0.721 & $49.0 \%$ \\
\hline
\end{tabular}

the result is also analyzed. The single run time of the program is 58 minutes and 36 seconds. The results are shown in Tables 3 and 4.

It can be seen from Tables 3 and 4 that for the SA and UA that are designed under the precondition of equal gain loss, the R-PSLL is $1.937 \mathrm{~dB}\left(\varphi=0^{\circ}\right.$ plane $) / 1.586 \mathrm{~dB}\left(\varphi=90^{\circ}\right.$ plane) lower than that of UA. Besides, the average PSLL of SA is $1.402 \mathrm{~dB}\left(\varphi=0^{\circ}\right) / 1.406 \mathrm{~dB}\left(\varphi=90^{\circ}\right)$ lower than that of $\mathrm{UA}$. What is more, the ratio of $\triangle \mathrm{PSLL} \leq 1 \mathrm{~dB}$ of SA is very close to $100 \%$ and is almost twice that of UA, which indicates that the position tolerance of SA basically guarantees the requirements for the gain and the PSLL of SA.

Besides, another verification work is done in order to give a comprehensive view of the new method. Suppose the requirement for the array is the allowable increasement of the peak sidelobe level $\triangle$ PSLL $_{\mathrm{a}} \leq 1 \mathrm{~dB}$, then the preset requirement of SA and $\mathrm{UA}$ is $\triangle \mathrm{PSLL}_{\mathrm{a}}^{\mathrm{S}}=\Delta \mathrm{PSLL}_{\mathrm{a}}^{\mathrm{U}}=1 \mathrm{~dB}$ and the R-GL of SA and UA is compared. Here the way to obtain the relationship $\triangle \mathrm{PSLL}=f(\mathrm{~T})$ for SA is similar to the way to obtain GL $=f(\mathbf{T})$ that is shown in Figure 4, except that the position tolerance $T_{a}^{S}$ is obtained based on the preset requirements for $\triangle \mathrm{PSLL}_{\mathrm{a}}$ while the latter is based on the preset requirements for GL. The UA is designed by the method proposed by Elliott [23] who deduced relationship between the PSLL and the position tolerance $T_{a}^{U}$. The reliable group number $X_{r}=5000$ when the variation range of $\triangle \mathrm{PSLL}$ is $0.1 \mathrm{~dB}$. The result is shown in Tables 5 and 6 .

It can be seen from Tables 5 and 6 that the mean PSLL of SA is $0.749 \mathrm{~dB}\left(\varphi=0^{\circ}\right) / 0.726 \mathrm{~dB}\left(\varphi=90^{\circ}\right)$ lower than that of UA. Besides, although the R-GL and the mean gain loss are $0.285 \mathrm{~dB}$ and $0.252 \mathrm{~dB}$ higher than those of $\mathrm{UA}$, respectively, the mean position tolerance of UA $(0.025 \lambda)$ is 
TABLE 5: Comparison of electrical performance parameters between UA and SA (unit: $\mathrm{dB}$ ).

\begin{tabular}{lcccc}
\hline & R-GL & $\begin{array}{c}\text { R-PSLL } \\
\left(\varphi=0^{\circ}\right)\end{array}$ & $\begin{array}{c}\text { R-PSLL } \\
\left(\varphi=90^{\circ}\right)\end{array}$ & Tolerance \\
\hline SA & 0.421 & -24.452 & -24.436 & $0.021 \lambda \sim 0.150 \lambda$ \\
UA & 0.136 & -24.255 & -24.372 & $0.025 \lambda$ \\
(Ref. [23]) & & -0.197 & -0.064 & $/$ \\
Difference & 0.285 & -
\end{tabular}

Here the difference is the parameter of SA minus that of UA.

TABLE 6: Uncertainty analysis of SA and UA under the precondition of equal PSLL.

\begin{tabular}{lcccc}
\hline & & Mean & Std. & Ratio \\
\hline \multirow{2}{*}{ PSLL $\left(\varphi=0^{\circ}\right)$} & SA & -25.609 & 0.655 & $96.6 \%$ \\
& $\begin{array}{c}\text { UA } \\
\text { (Ref. [23]) }\end{array}$ & -24.860 & 0.474 & $87.2 \%$ \\
\hline $\begin{array}{l}\text { PSLL } \\
\left(\varphi=90^{\circ}\right)\end{array}$ & SA & -25.607 & 0.654 & $96.5 \%$ \\
\hline Gain loss & UA & -24.881 & 0.477 & $87.7 \%$ \\
& $\begin{array}{c}\text { Sef. [23]) } \\
\text { SA (Ref. }\end{array}$ & 0.441 & 0.029 & $97.5 \%$ \\
\hline
\end{tabular}

much lower than that of SA $(0.073 \lambda)$, which indicates that the small gain loss of UA is achieved by greatly improving the position accuracy of all antenna elements. What is more, it can be seen from Tables 5 and 6 that the gain loss of SA has met the restriction for the requirements of $\mathrm{GL} \leq 0.5 \mathrm{~dB}$. Therefore, the SA designed through the new method is able to realize the desired performance through a more economic way. The above results fully demonstrate the effectiveness and the novelty of the new method proposed in this paper.

\section{Conclusion}

The high performance of array antenna guarantees the effective communication and accurate acquisition of target information in the Internet of Things. The position tolerance design of array antenna is one of the important research areas. In this paper, the sensitivity model of the array radiation field to antenna element position is derived, and a novel method for array tolerance design is proposed based on the position sensitivity. Compared with the array designed by the "traditional method," the desired electrical performance of the newly designed array can be better achieved through a more economic way, which demonstrated the innovation and effectiveness of the new method. Besides, the sensitivity model can also be used for the theoretical basis of structural strength design. In a word, the study in this paper provides theoretical guidance for the implementation of Internet of Things.

\section{Conflicts of Interest}

The authors declare that they have no conflicts of interest.

\section{Acknowledgments}

This work was supported by the National Natural Science Foundation of China under Grant nos. 51522507 and 51475349, the National 973 Program under Grant no. 2015CB857100, Youth Science and Technology Star Project of Shaanxi Province under Grant no. 2016KJXX-06, the Fundamental Research Funds for the Central Universities under Grant nos. JBG150409, KJXX1603, and 7214479606.

\section{References}

[1] V. Beltran, A. F. Skarmeta, and P. M. Ruiz, "An ARM-compliant architecture for user privacy in smart cities: SMARTIE-quality by design in the IoT," Wireless Communications and Mobile Computing, vol. 2017, Article ID 3859836, 13 pages, 2017.

[2] D. Wang, H. Liu, X. Ma, J. Wang, Y. Peng, and Y. Wu, "Energy harvesting for internet of things with heterogeneous users," Wireless Communications and Mobile Computing, vol. 2017, Article ID 1858532, 15 pages, 2017.

[3] L. Atzori, A. Iera, and G. Morabito, The Internet of Things: A survey, Elsevier North-Holland Inc, 2010.

[4] S. Andreev, S. Balandin, and Y. Koucheryavy, Internet of Things, Smart Spaces, and Next Generation Networks and Systems, Springer, 2015.

[5] K. Yelamarthi, M. S. Aman, and A. Abdelgawad, "An application-driven modular IoT architecture," Wireless Communications and Mobile Computing, vol. 2017, Article ID 1350929, 16 pages, 2017.

[6] S. F. Fang, L. D. Xu, Y. Q. Zhu et al., "An integrated system for regional environmental monitoring and management based on internet of things," IEEE Transactions on Industrial Informatics, vol. 10, no. 2, pp. 1596-1605, 2014.

[7] V. Mohanan, R. Budiarto, and I. Aldmour, Powering the Internet of Things with $5 G$ Networks, IGI Global, 2017.

[8] W. M. Ibrahim, N. A. Ali, H. S. Hassanein, and A.-E. M. Taha, "Characterizing multi-hop localization for Internet of things," Wireless Communications and Mobile Computing, vol. 2016, 16 pages, 2016.

[9] R. Fan, H. Jiang, S. Wu, and N. Zhang, "Ranging error-tolerable localization in wireless sensor networks with inaccurately positioned anchor nodes," Wireless Communications Mobile Computing, vol. 9, 13 pages, 2009.

[10] J. Bernhard, Phased Array Antenna Handbook, Artech House, 1994.

[11] H. Schippers, G. Spalluto, and G. Vos, "Radiation analysis of conformal phased array antennas on distorted structures," in Proceedings of the Twelfth International Conference on Antennas and Propagation (ICAP 2003), pp. 160-163, 2003.

[12] C. S. Wang, B. Y. Duan, and Y. Y. Qiu, "On distorted surface analysis and multidisciplinary structural optimization of large reflector antennas," Structural and Multidisciplinary Optimization, vol. 33, no. 6, pp. 519-528, 2007.

[13] J. Zhou, L. Song, J. Huang, and C. Wang, "Performance of structurally integrated antennas subjected to dynamical loads," International Journal of Applied Electromagnetics and Mechanics, vol. 48, no. 4, pp. 409-422, 2015.

[14] C. S. Wang, B. Y. Duan, F. S. Zhang, and M. B. Zhu, "Coupled structural-electromagnetic-thermal modelling and analysis of active phased array antennas," IET Microwaves, Antennas \& Propagation, vol. 4, no. 2, pp. 247-257, 2010. 
[15] E. Zaitsev and J. Hoffman, "Phased array flatness effects on antenna system performance," in Proceedings of the 4th IEEE International Symposium on Phased Array Systems and Technology, Array 2010, pp. 121-125, Waltham, MA, USA, October 2010.

[16] B. Y. Duan and C. S. Wang, "Reflector antenna distortion analysis using MEFCM," IEEE Transactions on Antennas and Propagation, vol. 57, no. 10, pp. 3409-3413, 2009.

[17] J. Ruze, "The effect of aperture errors on the antenna radiation pattern," Il Nuovo Cimento, vol. 9, no. 3, pp. 364-380, 1952.

[18] J. K. Hsiao, "Array sidelobes, error tolerance, gain, and beamwidth," Tech. Rep. Nrl-8841, Naval Research Lab, Washington, DC, USA, 1984.

[19] P. Rocca, L. Manica, N. Anselmi, and A. Massa, "Analysis of the pattern tolerances in linear arrays with arbitrary amplitude errors," IEEE Antennas and Wireless Propagation Letters, vol. 12, pp. 639-642, 2013.

[20] L. Poli, P. Rocca, N. Anselmi, and A. Massa, "Dealing With Uncertainties on Phase Weighting of Linear Antenna Arrays by Means of Interval-Based Tolerance Analysis," IEEE Transactions on Antennas and Propagation, vol. 63, no. 7, pp. 3229-3234, 2015.

[21] L. L. B. Bailin and M. J. Ehrlich, "Factors Affecting the Performance of Linear Arrays," Proceedings of the IRE, vol. 41, no. 2, pp. 235-241, 1953.

[22] H. S. C. Wang, "Performance of Phased-Array Antennas with Mechanical Errors," IEEE Transactions on Aerospace and Electronic Systems, vol. 28, no. 2, pp. 535-545, 1992.

[23] R. Elliott, "Mechanical and electrical tolerances for twodimensional scanning antenna arrays," IRE Transactions on Antennas Propagation, vol. 6, no. 1, pp. 114-120, 1958.

[24] M. Lanne, "Design aspects and pattern prediction for phased arrays with subarray position errors," in Proceedings of the 4th IEEE International Symposium on Phased Array Systems and Technology, Array 2010, pp. 440-446, Waltham, MA, USA, October 2010.

[25] C. Wang, M. Kang, W. Wang et al., "Electromechanical coupling based performance evaluation of distorted phased array antennas with random position errors," International Journal of Applied Electromagnetics and Mechanics, vol. 51, no. 3, pp. 285295, 2016.

[26] C. Wang, M. Kang, W. Wang, B. Duan, L. Lin, and L. Ping, “On the performance of array antennas with mechanical distortion errors considering element numbers," International Journal of Electronics, vol. 104, no. 3, pp. 462-484, 2017.

[27] A. Saltelli, Global Sensitivity Analysis: The Primer, 2008.

[28] R. Levine and D. Renelt, "A sensitivity analysis of cross-country growth regressions," American Economic Review, vol. 82, no. 4, pp. 942-963, 1992.

[29] V. Komkov, K. C. Kyung, and J. H. Edward, Design Sensitivity Analysis of Structural Systems, vol. 177, Academic press, 1986.

[30] S. Zhang, B. Duan, H. Bao, and P. Lian, "Sensitivity analysis of reflector antennas and its application on shaped geo-truss unfurlable antennas," IEEE Transactions on Antennas and Propagation, vol. 61, no. 11, pp. 5402-5407, 2013.

[31] G. F. Carrier, M. Krook, and C. E. Pearson, "Functions of a complex variable - theory and technique," American Mathematical Monthly, vol. 2, pp. 221-222, 2005.

[32] J. F. Kenney, Mathematics of Statistics, New Delhi, India, D. Van Nostrand Company Inc, London, UK; Affiliated East-West Press Pvt-Ltd, 2013. 


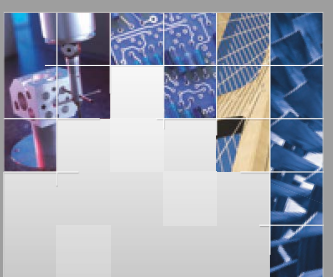

\section{Enfincering}
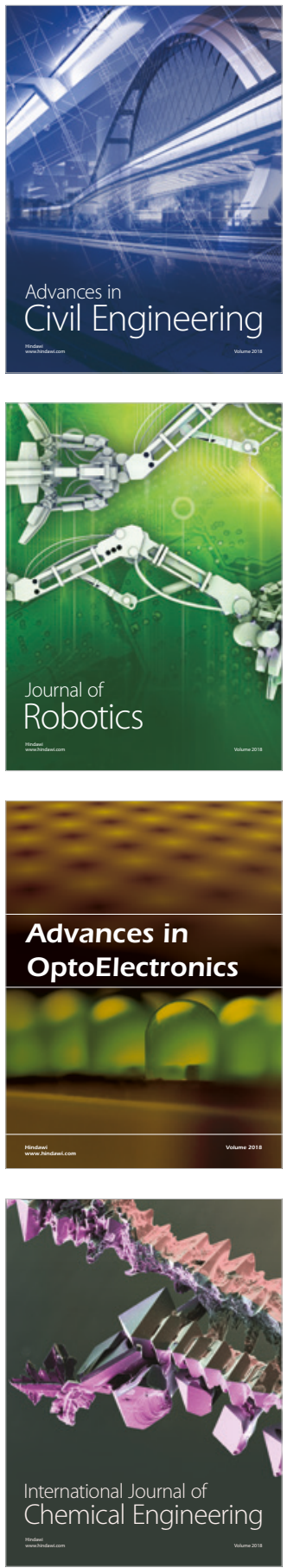

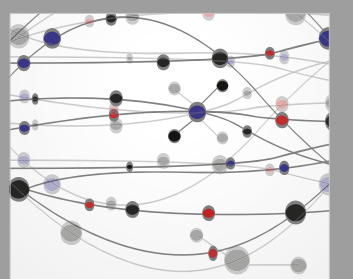

\section{Rotating \\ Machinery}

The Scientific World Journal

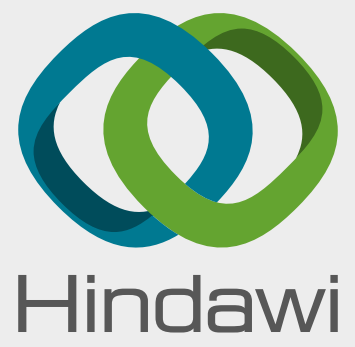

Submit your manuscripts at

www.hindawi.com
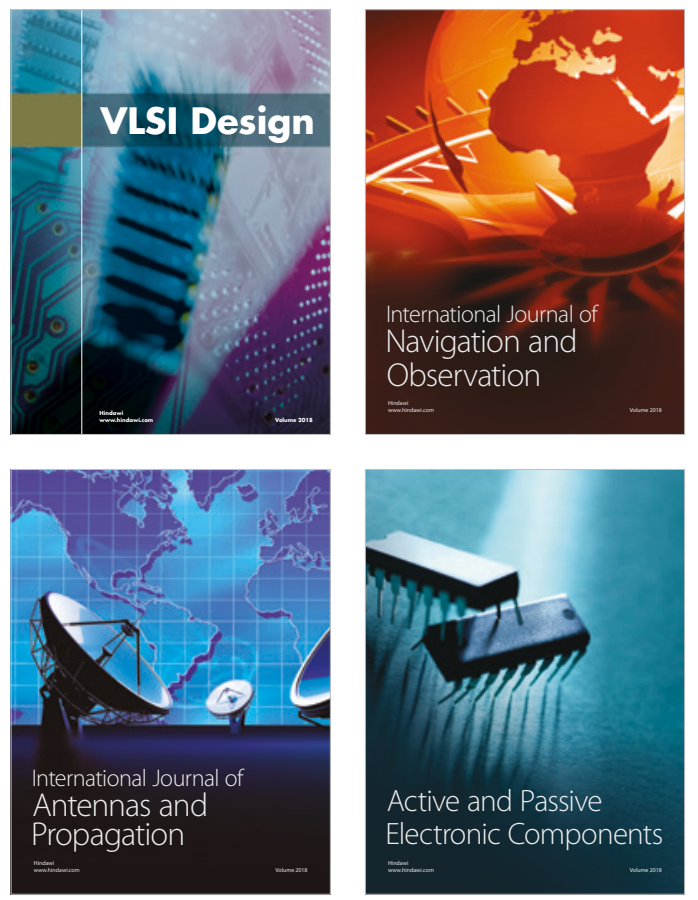
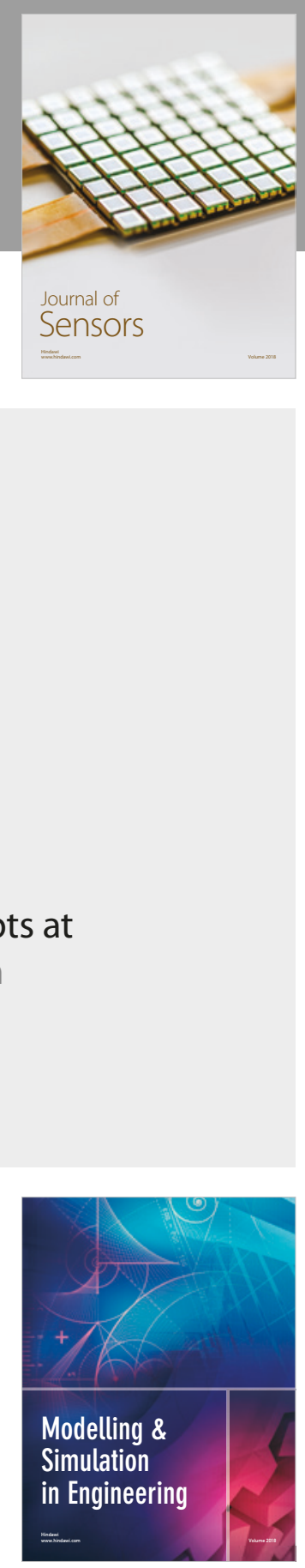

\section{Advances \\ Multimedia}
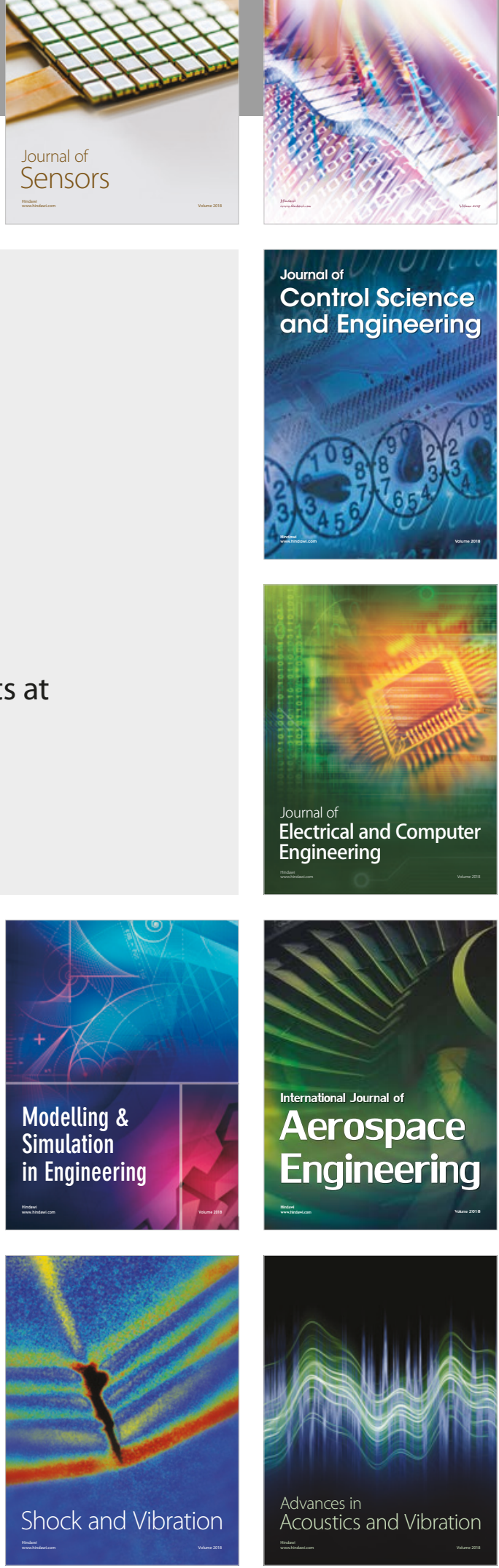